\title{
Impact of mode of conception on neonatal and neurodevelopmental outcomes in preterm infants
}

\author{
L Molines ${ }^{1}$, S Nusinovici'2,3, M Moreau¹, M Remy ${ }^{1}$, P May-Panloup ${ }^{1}$, C Flamant ${ }^{2,3}$, JC Roze ${ }^{2,3}$, P Van \\ Bogaert ${ }^{1,2}$, PE Bouet ${ }^{1}$, G Gascoin ${ }^{1,2}$ \\ ${ }^{1}$ Angers University Hospital, Angers, France \\ 2 Loire Infant Follow-Up Team (LIFT) Network, Pays de Loire, France \\ ${ }^{3}$ Nantes University Hospital, Nantes, France

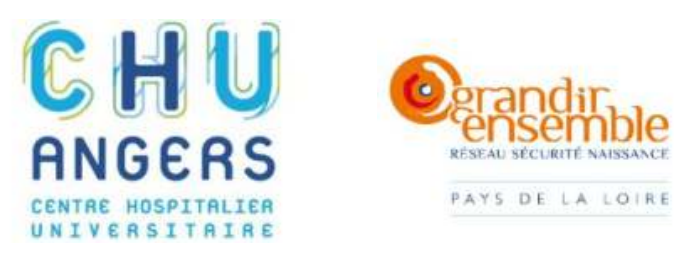

Objective: Assisted conception appears to increase the rate of preterm births, though few studies analysed outcomes for these preterm infants. The objective of this study was to assess the effect of assisted conception on neonatal morbidity and mortality and on neurodevelopmental outcome at 2-years in preterm infants born before 34 weeks of gestational age.

Methods: We conducted a single-center cohort study. All infants born alive between $24+0$ and $33+6$ weeks of gestational age and hospitalised at the Angers University Hospital were eligible as long as the mode of conception was known for neonatal outcome assessment. Live infants at discharge were enrolled in the Loire Infant Follow-up Team (LIFT) prospective longitudinal cohort and included for neurodevelopmental outcome assessment. Neonatal morbidity and mortality were evaluated during hospitalisation based on a composite score including death, intraventricular haemorrhage grade $\geq 3$, periventricular leukomalacia, treated patent ductus arteriosus and bronchopulmonary dysplasia at 36 weeks of gestational age. The neurodevelopmental outcome at 2 -years of corrected age was appreciated by a physical examination, a neuropsychological test and a parental questionnaire. In order to ensure comparability, infants were matched 1:1 according to maternal age, twin status and propensity score to be born after assisted conception including gestational age, z-score of birth weight, antenatal corticosteroids and magnesium sulphate treatments, gender, parity, maternal body mass index, tobacco consumption, outborn and maternal socio-economic status.

Results: 703 preterm infants were included in the analysis of neonatal morbidity and mortality, including 137 born after assisted conception. After matching, 184 preterm infants were included for neonatal morbidity and mortality analysis. There was no significant association between assisted conception and neonatal morbidity and mortality $(\mathrm{aOR} 0.67,95 \% \mathrm{Cl}[0.25,1.77], \mathrm{p}=0.422)$. 573 infants were assessed at 2 years, including 121 born after assisted conception. After matching, 154 preterm infants were included for neurodevelopmental outcome analysis. Assisted conception was significantly associated with a reduction in the probability of non-optimal neurological development at 2 years (aOR $0.26,95 \% \mathrm{Cl}[0.09,0.80], p=0.019)$.

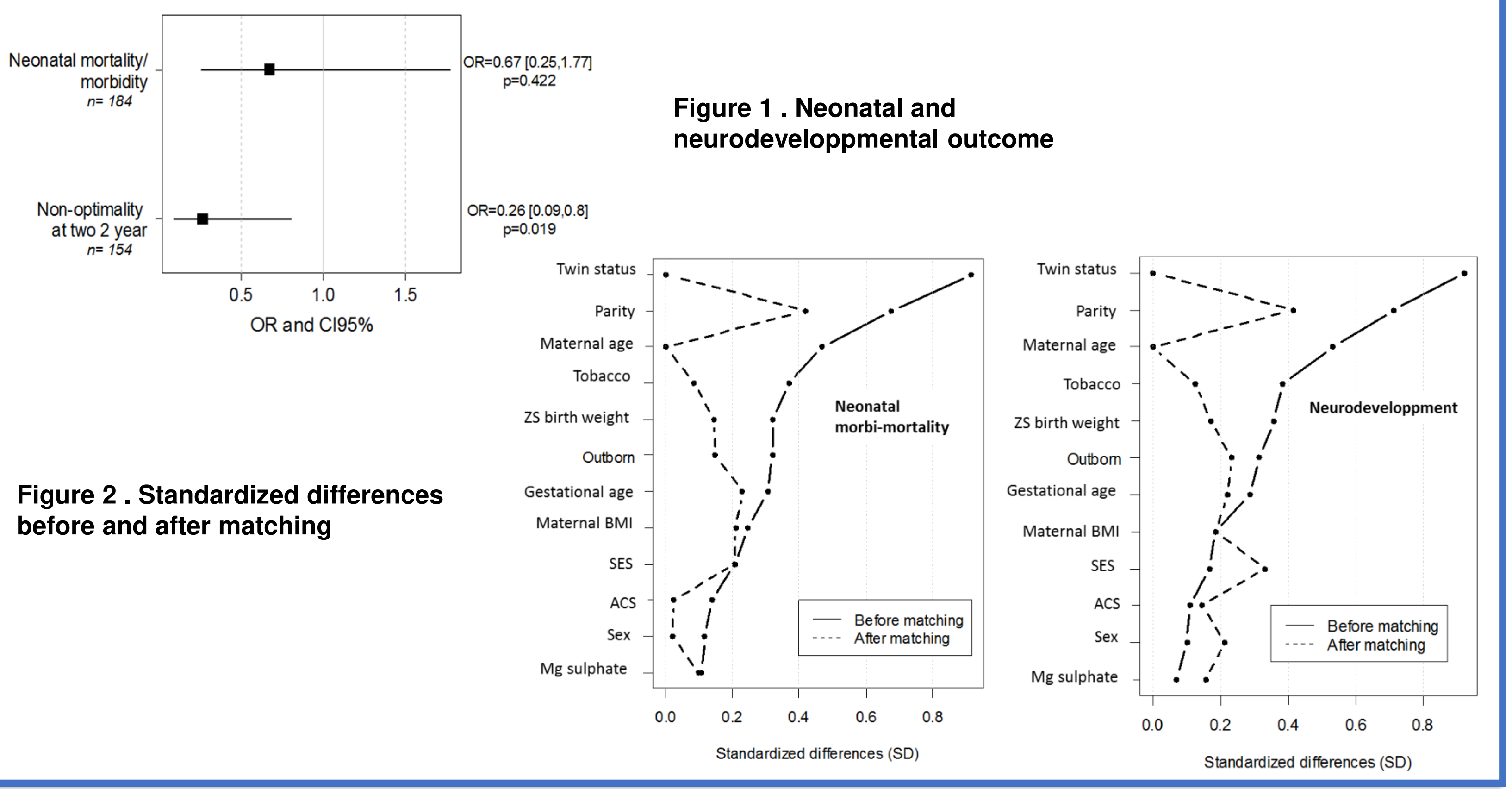

\section{Discussion:}

- Regarding the possible effect between assisted conception and neonatal morbidity, our study did not allow to conclude whether there is no effect of assisted or if the sample size is too low; therefore, further research with larger sample size is needed. Nevertheless our results are consistent with most previous studies.

- Only one study has evaluated neurodevelopment at 2-3 years corrected age in infants conceived by assisted conception, though these were more immature than in the population used for our study as they were born at less than 29 weeks GA (Abdel-Latif et al., 2013). The proportion of infants born after assisted conception with a functional disability was comparable to that of naturally spontaneously conceived infants. Our results are not in agreement with this study. A possible explanation for this could be the closer follow-up of infants often long-desired and awaited by their parents, along with a more favorable living environment.

We did not find any significant association between assisted conception and neonatal morbidity and mortality in preterm infants born before 34 weeks of gestational age.

Moreover, assisted conception was significantly associated with a reduced probability of non-optimal psychomotor development at 2 years of corrected age. 